\title{
Superconductivity, Charge Density Wave and Charge Kondo States in Systems of Coexisting Itinerant Electrons and Local Pairs
}

\author{
M. Sidowski AND S. RobASZKIEWICZ \\ Institute of Physics, A. Mickiewicz University \\ Umultowska 85, 61-614 Poznań, Poland
}

\begin{abstract}
The properties of a system of coexisting local pairs and itinerant electrons described by the (hard-core) boson-fermion model are discussed. For the first time we include into analysis of the model not only the superconducting and non-ordered (normal) states but also the charge density wave phases as well as the so-called charge Kondo state. Within an extended mean-field approximation, a mutual stability of charge density wave, superconducting and charge Kondo states are determined at $T=0$ in the case of half-filled fermionic and bosonic bands.
\end{abstract}

PACS numbers: 74.20.Mn, 74.20.-z, 75.30.Mb, 71.45.Lr

\section{Introduction}

The purpose of the present paper is analysis of the phase diagrams and electron orderings of a system of coexisting local electron pairs and itinerant electrons described by the (hard-core) boson-fermion model. The Hamiltonian of itinerant $c$ electrons and the $d$ electrons creating local pairs (LP) has the form

$$
\hat{H}=\sum_{i} \Delta_{0} n_{i}^{d}+\sum_{\langle i j\rangle \sigma} t_{i j} c_{i \sigma}^{+} c_{j \sigma}+I_{0} \sum_{i}\left(c_{i \uparrow}^{+} c_{i \downarrow}^{+} \rho_{i d}^{-}+\text {h.c. }\right)+\frac{1}{2} V_{0} \sum_{i} n_{i}^{d} n_{i}^{c} .
$$

The model takes into account both the intersubsystem charge coupling $I_{0}$ as well as the density-density interaction $V_{0}, n_{i}^{c}=n_{i \uparrow}^{c}+n_{i \downarrow}^{c}, n_{i}^{d}=n_{i \uparrow}^{d}+n_{i \downarrow}^{d}$, a total number of particles per site is $n=n_{c}+n_{d}=\left(\sum_{i}\left\langle n_{i}^{c}\right\rangle+\sum_{i}\left\langle n_{i}^{d}\right\rangle\right) / N . \Delta_{0}-D$ measures the relative position of the LP level with respect to the bottom of $c$-electron band, $2 D=2 z t$ is the band width of $c$-electron band in the absence of interactions, $z$ is the number of nearest neighbours (nn). The Hamiltonian (1) is defined in the subspace excluding single occupancy of sites by $d$ electrons. In this subspace the charge operators $\left\{\boldsymbol{\rho}_{i d}\right\}$, defined by $\rho_{i d}^{+}=d_{i \uparrow}^{+} d_{i \downarrow}^{+}=\left(\rho_{i d}^{-}\right)^{+}, \rho_{i d}^{z}=\frac{1}{2}\left(n_{i d}-1\right)$, obey the Pauli spin $\frac{1}{2}$ commutation relations and the following relations are fulfilled: 


$$
n_{i \uparrow}^{d} n_{i \downarrow}^{d}=\rho_{i d}^{z}+\frac{1}{2}, \quad\left(n_{i \uparrow}^{d}-n_{i \downarrow}^{d}\right)^{2}=0 .
$$

Up to now the studies of the model have been concentrated on the superconducting (SC) and non-ordered (normal) phases [1-5]. We extend the previous investigation and include into the analysis the charge density wave (CDW) phases as well as the so-called charge Kondo state (CKS). The CKS being an analogue of the magnetic Kondo state in the systems of the periodic Kondo lattice is characterized by a compensation of a local charge moment (isospin singlet) $[1,4-6]$. One finds that such a state can be realized in the present model if the intersubsystem charge exchange interaction is increased, and it can compete with SC and CDW orderings.

We have performed a detailed analysis of the phase diagrams and thermodynamic properties of the model (1) for $d$-dimensional hypercubic lattices and arbitrary, positive and negative $I_{0}$ and $V_{0}[6]$. In the analysis we have used an extended mean-field approximation (MFA-HFA), analogous to that used in the treatment of the Kondo lattice model [7, 8]. Below we only quote the main results of this investigation, concentrating on the case of half-filled bands: $n_{c}=n_{d}=1$ which is realized for $n=2$ and $\Delta_{0}=0$. We will restrict our analysis to the pure phases and assume rectangular density of states (DOS) for $c$-electron band.

Definitions of the phases considered and the corresponding order parameters are the following:

superconducting phase (SC) $\rho_{0}^{x} \neq 0, x_{0} \neq 0$, where

$$
\rho_{0}^{x}=\frac{1}{2 N} \sum_{i}\left\langle\rho_{i d}^{+}\right\rangle \text {and } x_{0}=\frac{1}{N} \sum_{k}\left\langle c_{k \uparrow}^{+} c_{-k \downarrow}^{+}\right\rangle,
$$

charge-ordered phase (CDW) $\rho_{Q}^{z} \neq 0, n_{Q} \neq 0$, where

$$
\begin{aligned}
& \rho_{Q}^{z}=\frac{1}{N} \sum_{i}\left\langle n_{i}^{d}\right\rangle \mathrm{e}^{-\mathrm{i} \boldsymbol{Q} \boldsymbol{R}_{\mathrm{i}}}=\frac{1}{N} \sum_{i}\left\langle 2 \rho_{i d}^{z}\right\rangle \mathrm{e}^{-\mathrm{i} \boldsymbol{Q} \boldsymbol{R}_{\mathrm{i}}}, \quad n_{Q}=\frac{1}{N} \sum_{k \sigma}\left\langle c_{k+Q \sigma}^{+} c_{k \sigma}\right\rangle, \\
& \boldsymbol{Q}=\left(\frac{\pi}{a}, \frac{\pi}{a}, \frac{\pi}{a}\right),
\end{aligned}
$$

charge Kondo state (CKS)

$$
\lambda=\frac{1}{2 N} \sum_{i}\left(\left\langle d_{i \sigma}^{+} c_{i \sigma}\right\rangle+\text { h.c. }\right) .
$$

\section{Results and discussion}

For $n=2$ and $\Delta_{0}=0$ one finds that the chemical potential $\mu=V_{0} / 2$ and $n_{c}=n_{d}=1$ for any $T$. In such a case the ground state diagram of the model (1), taking into account only pure phases and calculated for rectangular DOS, is plotted in Fig. 1.

In Figs. 2-4 we present the evolution of the order parameters and the quasiparticle gap in the excitation spectrum $E_{\mathrm{g}}$ at $T=0$ with increasing interactions. 


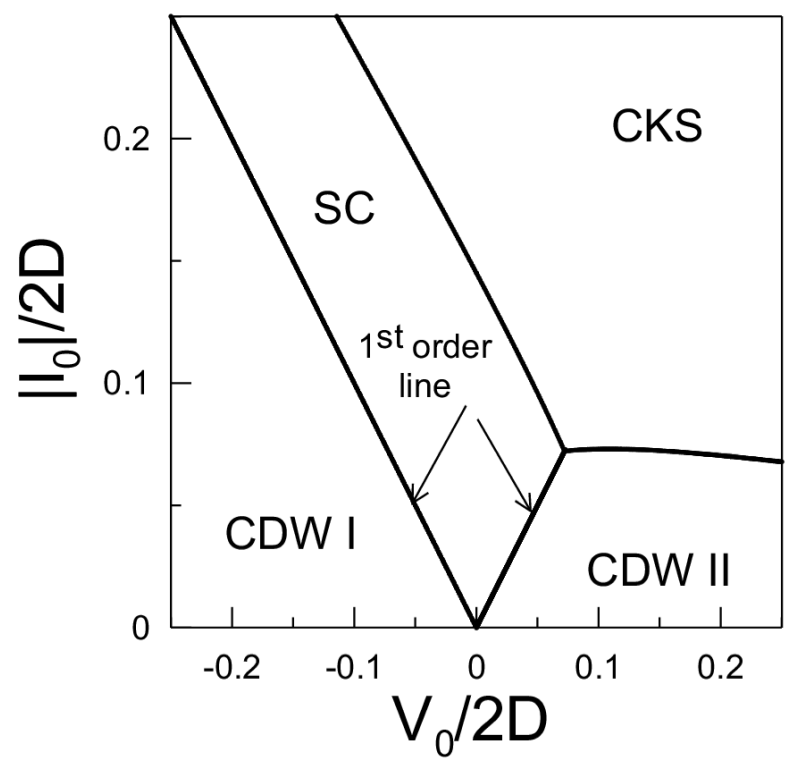

Fig. 1. Ground state phase diagram of the model (1) at half-filling plotted as a function of $I_{0} / 2 D$ and $V_{0} / 2 D$, for $n=2, \Delta_{0} / 2 D=0, n_{c}=n_{d}=1$, for rectangular DOS.

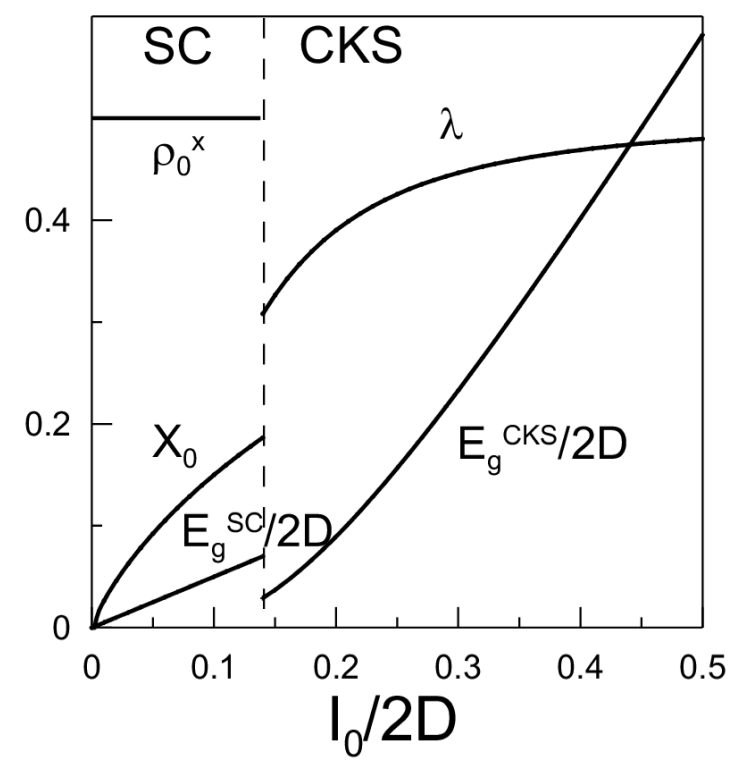

Fig. 2. Variation of the SC and CKS order parameters and the quasiparticle gap at $T=0$ as a function of $I_{0} / 2 D$ for $n=2, \Delta_{0} / 2 D=0, V_{0} / 2 D=0$. 


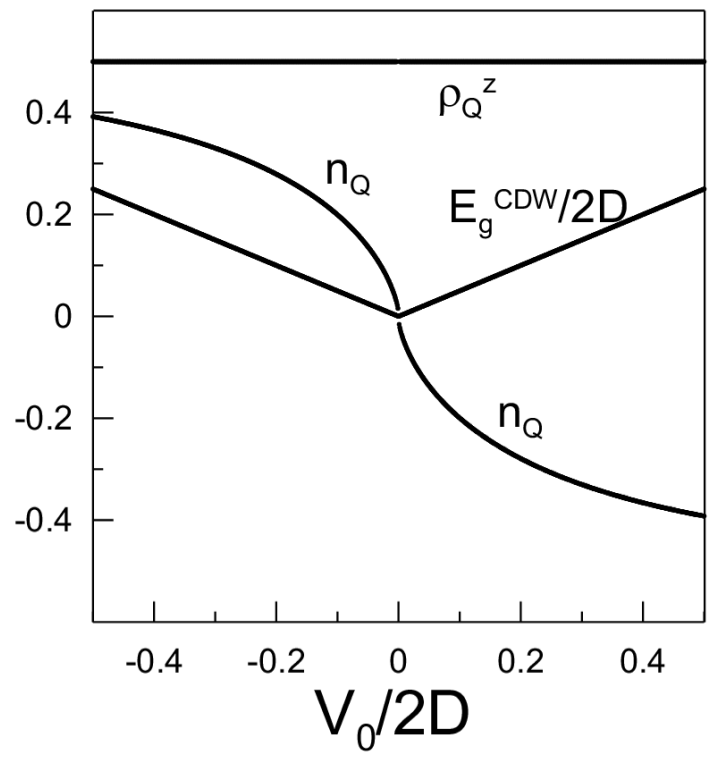

Fig. 3. Variation of the CDW order parameter $\rho_{Q}^{z}$ and $n_{Q}$ and the quasiparticle gap $E_{\mathrm{g}}^{\mathrm{CDW}} / 2 D$ at $T=0$ as a function of $V_{0} / 2 D$ for $n=2, \Delta_{0} / 2 D=0, I_{0} / 2 D=0$.

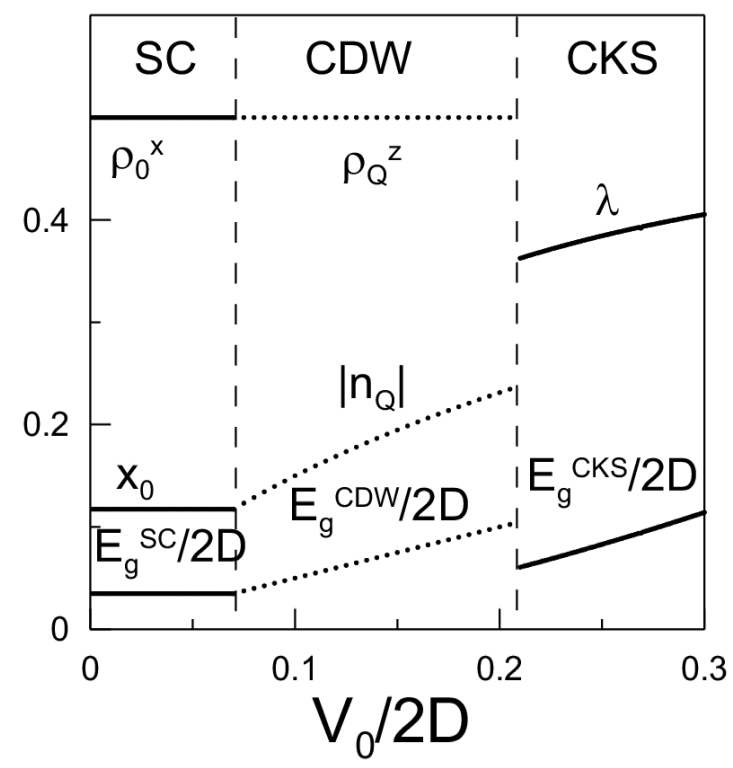

Fig. 4. Variation of the SC, CDW and CKS order parameters and the quasiparticle gaps at $T=0$ as a function of $V_{0} / 2 D$ for $n=2, \Delta_{0} / 2 D=0, I_{0} / 2 D=0.07$. 


\section{Summary}

Let us shortly summarize our main findings.

1. For small values of $I_{0} / 2 D$ and $V_{0} / 2 D$ the ground state is SC, if $\left|I_{0}\right|>\left|V_{0}\right|$ and CDW if $\left|I_{0}\right|<\left|V_{0}\right|$, for both signs of $I_{0}$ and $V_{0}$ (cf. Fig. 1).

2. For $\left|I_{0}\right|>\left|V_{0}\right|$ and $T=0$, with increasing $\left|I_{0}\right| / 2 D$ the system exhibits a transition from SC to CKS state at $\left(\left|I_{0}\right| / 2 D\right)_{c}$ (cf. Figs. 1 and 2). The value of $\left(\left|I_{0}\right| / 2 D\right)_{c}$ depends on the lattice structure of the system and the strength of $V_{0}$ : repulsive $V_{0}\left(V_{0}>0\right)$ reduces this critical value whereas attractive $V_{0}\left(V_{0}<0\right)$ enhances it.

3. For $\left|I_{0}\right|<\left|V_{0}\right|$ and $T=0$ the system remains in the CDW state for any $\left|V_{0}\right| / 2 D$, if $V_{0}<0$, whereas for repulsive $V_{0}\left(V_{0}>0\right)$ the increase in $\left|I_{0}\right| / 2 D$ yields a transition $\mathrm{CDW} \rightarrow \mathrm{CKS}$, and the critical value $\left(\left|I_{0}\right| / 2 D\right)_{c}$ slowly decreases with increasing $V_{0} / 2 D$ (cf. Fig. 1).

4. The phases CDW and CKS are nonmetallic and at the borders of these phases with SC the system exhibits the nonmetal-superconductor transition.

5. The CDW ordering is stabilized by the intersubsystem density interaction $V_{0}$ (both the repulsive and attractive one). It involves spatial modulation of charge in both subsystems and the order parameters $\rho_{Q}^{z}$ and $n_{Q}$ have the same signs for $V_{0}<0$ and the opposite signs for $V_{0}>0$, which maximizes (minimizes) the total amplitude of charge modulation for $V_{0}<0\left(V_{0}>0\right)$ (cf. Fig. 3) and we call the CDW phases realized for $V_{0}<0$ and $V_{0}>0$ as CDW I and CDW II, respectively.

6. The plots of CDW order parameters and the gap in the $c$-electron spectrum at $T=0$ as a function of $\left|V_{0}\right| / 2 D$ for $\left|I_{0}\right| / 2 D=0$ are shown in Fig. 2.

7. With increasing $V_{0} / 2 D\left(-\infty<V_{0} / 2 D<\infty\right)$ for a fixed value of $I_{0} / 2 D$ the system can exhibit the following sequences of transitions: CDW I $\rightarrow \mathrm{SS} \rightarrow$ $\mathrm{CDW}$ II $\rightarrow$ CKS, if $0<I_{0} / 2 D<\left(I_{0} / 2 D\right)_{c}$, and $\mathrm{CDW} \mathrm{I} \rightarrow \mathrm{SS} \rightarrow \mathrm{CKS}$, if $I_{0} / 2 D>\left(I_{0} / 2 D\right)_{c}$.

At present we study the case of arbitrary particle concentrations and include into consideration the mixed ordered phases. Our preliminary results clearly indicate that the deviation from the half-filling (i.e. from $n_{c}=n_{d}=1$ ) with a change of $n$ or/and $\Delta_{0}$ can strongly extend the range of stability of SC phase with respect to other phases.

\section{Acknowledgments}

We thank R. Micnas for helpful discussion. This work was supported in part by the State Committee for Scientific Research, grant No. 1 P03B 084 26, and by the Foundation for Polish Science. 


\section{References}

[1] S. Robaszkiewicz, R. Micnas, J. Ranninger, Phys. Rev. B 36, 180 (1987); J. Ranninger, S. Robaszkiewicz, Physica B 135, 468 (1985).

[2] R. Micnas, J. Ranninger, S. Robaszkiewicz, Rev. Mod. Phys. 62, 113 (1990) and references therein.

[3] R. Micnas, S. Robaszkiewicz, A. Bussmann-Holder, Phys. Rev. B 66, 104516 (2002) and references therein.

[4] A. Taraphder, P. Coleman, Phys. Rev. Lett. 66, 2814 (1991).

[5] S. Robaszkiewicz, Acta Phys. Pol. A 85, 117 (1994).

[6] M. Sidowski, S. Robaszkiewicz, in preparation.

[7] C. Lacroix, M. Cyrot, Phys. Rev. B 20, 1969 (1979).

[8] A.R. Ruppenthal, J.R. Iglesias, M.A. Gusmão, Phys. Rev. B 60, 7321 (1999). 